\title{
Angular Decomposition and Sign Solved Propagator of the 2D and 3D Helium Atom Path Integrals
}

\section{Emmanouil George Thrapsaniotis}

Athens, Greece

Email: egthra@hotmail.com

How to cite this paper: Thrapsaniotis, E.G. (2022) Angular Decomposition and Sign Solved Propagator of the 2D and 3D Helium Atom Path Integrals. Journal of Applied Mathematics and Physics, 10, 538-557. https://doi.org/10.4236/jamp.2022.102040

Received: January 21, 2022

Accepted: February 22, 2022

Published: February 25, 2022

Copyright $\odot 2022$ by author(s) and Scientific Research Publishing Inc. This work is licensed under the Creative Commons Attribution International License (CC BY 4.0).

http://creativecommons.org/licenses/by/4.0/

\begin{abstract}
In the present paper on the one hand we apply the central limit theorem to the solution of the sign problem of a path integral of two-interacting particles in potential and give an expression for the sign solved propagator (SSP) derived from that solution and on the other hand we perform the angular decomposition of the path integrals of the 2D and 3D Helium atoms. Finally, we combine those two results and derive the SSPs of the 2D and 3D Helium atoms.
\end{abstract}

\section{Keywords}

Propagator, Angular Decomposition, Sign Problem, Central Limit Theorem, 3D Helium, 2D Helium

\section{Introduction}

The central limit theorem has an enormous variety of applications in probability, statistics and various other areas of mathematics and mathematical physics [1] [2] [3].

Here we apply the central limit theorem to the solution of the sign problem [4] and the extraction of the sign solved propagator (SSP) [5] [6]. The sign problem [7] is a major problem in computational physics and quantum chemistry concerning the Monte Carlo evaluation of path integrals. Within all that context initially [4] we considered a toy example concerning multiple integrals and gave oscillation free equivalent expressions for their evaluation. Subsequently, we applied the whole approach to both phase space [5] and coherent spaces path integrals [6].

Portmanteau's theorem and Crammer's theorem [1] [2] have a central role 
within our method. According to Crammer's theorem let $X_{n}$ be a sequence of random variables and $\lambda_{n}$ constants with $\lim _{n \rightarrow \infty} \lambda_{n}=\infty$ such that for a constant $a$ $\lambda_{n}\left(X_{n}-a\right) \stackrel{D}{\rightarrow} X$. Moreover, let $f$ be a continuously differentiable function in a neighborhood of $a$. Then $\lambda_{n}\left(f\left(X_{n}\right)-f(a)\right) \stackrel{D}{\rightarrow} \dot{f}(a) X$. Therefore if $\sqrt{n}\left(X_{n}-\mu\right) \stackrel{D}{\rightarrow} Y$ where $Y$ is normally distributed with covariance $S$ and mean zero then $\sqrt{n}\left(f\left(X_{n}\right)-f(\mu)\right) \stackrel{D}{\rightarrow} X$ where $X$ is normally distributed with mean zero and covariance $\dot{f}^{\prime}(\mu) \mathrm{S} \dot{f}(\mu)$. So after such manipulation, we can use Portmanteau's theorem to switch to integral expressions.

Moreover, we have studied the case of the angular decomposition of path integrals [8].3D path integrals with spherical symmetry have the form

$$
K\left(\vec{r}_{f}, \vec{r}_{i} ; t\right)=\lim _{N \rightarrow \infty} \prod_{n=1}^{N} \int_{-\infty}^{\infty} \mathrm{d} \vec{r}_{n} \prod_{n=1}^{N+1} \int_{-\infty}^{\infty} \frac{\mathrm{d} \vec{p}_{n}}{(2 \pi)^{3}} \exp \left\{i \sum_{n=1}^{N+1}\left[\vec{p}_{n} \cdot\left(\vec{r}_{n}-\vec{r}_{n-1}\right)-\varepsilon\left(\frac{\vec{p}_{n}^{2}}{2}+V\left(\left|\vec{r}_{n}\right|\right)\right)\right]\right\}
$$

and angular decomposition

$$
K\left(\vec{r}_{f}, \vec{r}_{i} ; t\right)=\frac{1}{r_{f} r_{i}} \sum_{q=0}^{\infty} \sum_{m=-q}^{q} K_{q}\left(r_{f}, r_{i} ; t\right) Y_{q m}\left(\vartheta_{f}, \varphi_{f}\right) Y_{q m}^{*}\left(\vartheta_{i}, \varphi_{i}\right)
$$

where

$$
K_{q}\left(r_{f}, r_{i} ; t\right)=\int_{0}^{\infty} D r \int_{-\infty}^{\infty} \frac{D p}{2 \pi} \exp \left\{i \int_{0}^{t} \mathrm{~d} \tau\left[p \dot{r}-\left(\frac{p^{2}}{2}+\frac{q(q+1)}{2 r^{2}}+V(r)\right)\right]\right\}
$$

If the Hamiltonian of the path integrals includes angular terms we have to use the Faddeev-Popov delta function trick with respect to the angle and then perform standard manipulations (see Sections 3, 4).

In the present paper, we develop further those methods and consider double phase space path integrals of interacting particles. Further, we study the path integral of the $2 \mathrm{D}$ and $3 \mathrm{D}$ Helium atoms. We perform their angular decomposition, and then we derive their sign solved propagators (SSP).

The present paper proceeds as follows. In Section 2 we consider the application of the central limit theorem to a double path integral describing two interacting particles. We solve the sign problem and give the SSP. Then in Section 3, we consider the angular decomposition of the 3D Helium path integral and in Section 4 of the 2D one and apply the results of Section 2 to extract the SSPs. In Section 5, we give our conclusions. Finally, in the appendices, we give certain integrals appearing in Sections 3 and 4.

\section{Central Limit Theorem and Path Integrals}

We consider the double phase space path integral

$$
\begin{aligned}
K\left(r_{f}, r_{f}^{\prime} ; r_{i}, r_{i}^{\prime} ; t\right)= & \iiint \int \operatorname{Dr}(\tau) \frac{D p(\tau)}{2 \pi} D r^{\prime}(\tau) \frac{D p^{\prime}(\tau)}{2 \pi} h\left[r(\tau) ; r^{\prime}(\tau)\right] \\
& \times \exp \left[i \int_{0}^{t} \mathrm{~d} \tau\left(p(\tau) \dot{r}(\tau)+p^{\prime}(\tau) \dot{r}^{\prime}(\tau)-H_{0}\left(p, r ; p^{\prime}, r^{\prime} ; \tau\right)\right)\right]
\end{aligned}
$$


The discrete form of (4) composed of $N+1$ time slices is

$$
\begin{aligned}
& K^{(N)}\left(r_{f}, r_{f}^{\prime} ; r_{i}, r_{i}^{\prime} ; t\right) \\
& =\prod_{n=1}^{N}\left[\int \mathrm{d} r_{n}\right] \prod_{n=1}^{N+1}\left[\int \frac{\mathrm{d} p_{n}}{2 \pi}\right] \prod_{n=1}^{N}\left[\int \mathrm{d} r_{n}^{\prime}\right] \prod_{n=1}^{N+1}\left[\int \frac{\mathrm{d} p_{n}^{\prime}}{2 \pi}\right] \\
& \times h\left(r_{i}, r_{1}, r_{2}, \cdots, r_{N}, r_{f} ; r_{i}^{\prime}, r_{1}^{\prime}, r_{2}^{\prime}, \cdots, r_{N}^{\prime}, r_{f}^{\prime}\right) \\
& \times \exp \left\{i \sum_{n=1}^{N+1}\left[p_{n}\left(r_{n}-r_{n-1}\right)+p_{n}^{\prime}\left(r_{n}^{\prime}-r_{n-1}^{\prime}\right)-\varepsilon H_{0}\left(p_{n}, r_{n} ; p_{n}^{\prime}, r_{n}^{\prime} ; r_{n}\right)\right]\right\}
\end{aligned}
$$

We have set on the one hand $\varepsilon=\frac{t}{N+1}$ and $\tau_{n}=n \varepsilon$, and on the other $r_{0}=r_{i}$, $r_{0}^{\prime}=r_{i}^{\prime}, \quad r_{N+1}=r_{f}$, and $r_{N+1}^{\prime}=r_{f}^{\prime}$. In the present paper, we consider a Hamiltonian $H_{0}$ of the form

$$
H_{0}\left(p, r ; p^{\prime}, r^{\prime} ; \tau\right)=H(p, r)+H^{\prime}\left(p^{\prime}, r^{\prime}\right)+H_{I}\left(r, r^{\prime}\right)
$$

where

$$
H(p, r)=\frac{p^{2}}{2}+V(r, \tau)
$$

and

$$
H^{\prime}\left(p^{\prime}, r^{\prime}\right)=\frac{p^{\prime 2}}{2}+V^{\prime}\left(r^{\prime}, \tau\right)
$$

Other cases can be handled similarly.

Now we suppose that the integration variables in the multiple integral (5) are sampled by distributions, which for either the primed or the unprimed configuration space variables, are identical and statistically independent between them while the corresponding momentum space ones have densities which are the Fourier transform of the configuration space ones. Therefore on considering the central limit theorem [1] the following expressions of convergence in distribution applies

$$
\begin{gathered}
\sqrt{N+1}\left[\frac{1}{N+1} \sum_{n}\left[\begin{array}{c}
t \frac{p_{n}^{2}}{2} \\
t V\left(r_{n}, \tau_{n}\right)
\end{array}\right]-\left[\begin{array}{c}
t\left\langle\frac{p^{2}}{2}\right\rangle \\
t\langle V(r, t)\rangle
\end{array}\right]\right] \stackrel{D}{\longrightarrow} Z \\
\sqrt{N+1}\left[\frac{1}{N+1} \sum_{n}\left[\begin{array}{c}
t \frac{p_{n}^{\prime 2}}{2} \\
t V^{\prime}\left(r_{n}^{\prime}, \tau_{n}\right)
\end{array}\right]-\left[\begin{array}{c}
t\left\langle\frac{p^{\prime 2}}{2}\right\rangle \\
t\left\langle V^{\prime}\left(r^{\prime}, t\right)\right\rangle
\end{array}\right]\right] \stackrel{D}{\longrightarrow} Z^{\prime}
\end{gathered}
$$

For the time-dependent potential the mean value expression has the form

$$
\langle V(r, t)\rangle=\frac{1}{N+1} \sum_{n=1}^{N+1}\left\langle V\left(r_{n}, \tau_{n}\right)\right\rangle
$$

and similarly for the primed variables. In each term on the right-hand side of Equation (11) the expectation value is derived with respect to appropriate sampling distribution. The Gaussian random variables $Z$ and $Z^{\prime}$ are defined on the integration spaces. They obey normal probability density with mean zero 
and diagonal covariance matrices

$$
\vec{\Sigma}=\vec{\Sigma}_{2} \otimes I_{N+1}
$$

and

$$
\vec{\Sigma}^{\prime}=\vec{\Sigma}_{2}^{\prime} \otimes I_{N+1}
$$

respectively. The symbol $\otimes$ corresponds to the direct product of matrices and $I_{N+1}$ is the $N+1$-dimensional unit matrix. We have set

$$
\vec{\Sigma}_{2}=\left[\begin{array}{cc}
t^{2} \sigma_{m}^{2} & 0 \\
0 & t^{2} \sigma_{V}^{2}(t)
\end{array}\right]
$$

and

$$
\vec{\Sigma}_{2}^{\prime}=\left[\begin{array}{cc}
t^{2} \sigma_{m}^{\prime 2} & 0 \\
0 & t^{2} \sigma_{V}^{\prime 2}(t)
\end{array}\right]
$$

where

$$
\begin{aligned}
\sigma_{m}^{2} & =\operatorname{Var}\left(\frac{p^{2}}{2}\right) \\
\sigma_{V}^{2}(t) & =\operatorname{Var}(V(r, t)) \\
\sigma_{m}^{\prime 2} & =\operatorname{Var}\left(\frac{p^{\prime 2}}{2}\right) \\
\sigma_{V}^{\prime 2}(t) & =\operatorname{Var}\left(V^{\prime}\left(r^{\prime}, t\right)\right)
\end{aligned}
$$

The variances are calculated with respect to the same sampling distribution as in Equation (11). We can assume that the position and momentum random variables are statistically independent.

Therefore the distribution density of the random variable $Z$ in Equation (9) is

$$
f_{Z}(\vec{x})=\frac{1}{(2 \pi)^{N+1} \sqrt{\operatorname{det}(\vec{\Sigma})}} \exp \left[-\vec{x} \frac{1}{2 \vec{\Sigma}} \vec{x}^{\mathrm{T}}\right]
$$

where $\vec{x}=\left(p_{1}, p_{2}, \cdots, p_{N}, p_{N+1}, r_{1}, r_{2}, \cdots, r_{N}, r_{N+1}\right)$ and $\operatorname{det}(\vec{\Sigma})=\left(t^{4} \sigma_{m}^{2} \sigma_{V}^{2}(t)\right)^{N+1}$ is the determinant of the matrix $\vec{\Sigma}$. Similarly $Z^{\prime}$ has the distribution density

$$
f_{Z^{\prime}}\left(\vec{x}^{\prime}\right)=\frac{1}{(2 \pi)^{N+1} \sqrt{\operatorname{det}\left(\vec{\Sigma}^{\prime}\right)}} \exp \left[-\vec{x}^{\prime} \frac{1}{2 \vec{\Sigma}^{\prime}} \vec{x}^{\prime T}\right]
$$

where $\vec{x}^{\prime}=\left(p_{1}^{\prime}, p_{2}^{\prime}, \cdots, p_{N}^{\prime}, p_{N+1}^{\prime}, r_{1}^{\prime}, r_{2}^{\prime}, \cdots, r_{N}^{\prime}, r_{N+1}^{\prime}\right)$ and $\operatorname{det}\left(\vec{\Sigma}^{\prime}\right)=\left(t^{4} \sigma_{m}^{\prime 2} \sigma_{V}^{\prime 2}(t)\right)^{N+1}$.

We notice that in Equations (9), (10) we have sums of functions of independently distributed random vectors. Then according to an application of Lyapunov's theorem, for integrable functions with respect to the time, the domain of attraction is Gaussian and the above convergences in distribution apply.

Now we consider the functions

$$
f(x, y)=\cos (x+y)
$$




$$
g(x, y)=\sin (x+y)
$$

and apply a corollary of the Cramer's theorem (see in the introduction) in Equations (9), (10) to obtain the following convergence in distribution

$$
\sqrt{N+1}\left[\frac{1}{N+1} \sum_{n}\left[\begin{array}{c}
\cos \left(t H\left(p_{n}, r_{n}\right)\right) \\
\sin \left(t H\left(p_{n}, r_{n}\right)\right) \\
\cos \left(t H^{\prime}\left(p_{n}^{\prime}, r_{n}^{\prime}\right)\right) \\
\sin \left(t H^{\prime}\left(p_{n}^{\prime}, r_{n}^{\prime}\right)\right)
\end{array}\right]-\left[\begin{array}{c}
\cos (t\langle H(p, r)\rangle) \\
\sin (t\langle H(p, r)\rangle) \\
\cos \left(t\left\langle H^{\prime}\left(p^{\prime}, r^{\prime}\right)\right\rangle\right) \\
\sin \left(t\left\langle H^{\prime}\left(p^{\prime}, r^{\prime}\right)\right\rangle\right)
\end{array}\right]\right] \underset{D}{\longrightarrow} X
$$

The Gaussian random variable $X$ is defined on a space composed of $N+1$ copies of the system's space. Therefore as the domain of attraction is Gaussian it has a normal probability density with mean zero and diagonal covariance matrix

$$
t^{2} \operatorname{diag}\left[\sigma_{1}(t), \sigma_{2}(t), \sigma_{1}^{\prime}(t), \sigma_{2}^{\prime}(t)\right] \otimes I_{N+1}
$$

where $\sigma_{1}(t)=\sin ^{2}(t\langle H\rangle)\left(\sigma_{m}^{2}+\sigma_{V}^{2}(t)\right), \sigma_{2}(t)=\cos ^{2}(t\langle H\rangle)\left(\sigma_{m}^{2}+\sigma_{V}^{2}(t)\right)$, $\sigma_{1}^{\prime}(t)=\sin ^{2}\left(t\left\langle H^{\prime}\right\rangle\right)\left(\sigma_{m}^{\prime 2}+\sigma_{V}^{\prime 2}(t)\right), \quad \sigma_{2}^{\prime}(t)=\cos ^{2}\left(t\left\langle H^{\prime}\right\rangle\right)\left(\sigma_{m}^{\prime 2}+\sigma_{V}^{\prime 2}(t)\right)$. Notice that another way of looking at the above distribution is as a product of one-dimensional distributions.

Proceeding further we apply the above-mentioned corollary of Crammer's theorem for the functions

$$
\begin{aligned}
& f\left(x_{c}, x_{s}, x_{c}^{\prime}, x_{s}^{\prime}\right)=x_{c} x_{c}^{\prime}-x_{s} x_{s}^{\prime} \\
& g\left(x_{c}, x_{s}, x_{c}^{\prime}, x_{s}^{\prime}\right)=x_{c} x_{s}^{\prime}+x_{s} x_{c}^{\prime}
\end{aligned}
$$

and the central limit theorem (24) to obtain the final result

$$
\begin{aligned}
& \sqrt{N+1}\left[\exp \left[-i \varepsilon \sum_{n}\left(\frac{p_{n}^{2}}{2}+V\left(r_{n}, \tau_{n}\right)+\frac{p_{n}^{\prime 2}}{2}+V\left(r_{n}^{\prime}, \tau_{n}\right)\right)\right]\right. \\
& \left.-\exp \left[-i t\left(\langle H(t)\rangle+\left\langle H^{\prime}(t)\right\rangle\right)\right]\right] \stackrel{D}{\longrightarrow} Y-i W
\end{aligned}
$$

The random variables $Y, W$ are normal with mean zero and diagonal covariance matrices

$$
\begin{aligned}
& {\left[\sin ^{2}(t\langle H\rangle) \cos ^{2}\left(t\left\langle H^{\prime}\right\rangle\right)+\cos ^{2}(t\langle H\rangle) \sin ^{2}\left(t\left\langle H^{\prime}\right\rangle\right)\right] t^{2}\left(\sigma_{m}^{2}+\sigma_{V}^{2}(t)+\sigma_{m}^{\prime 2}+\sigma_{V}^{\prime 2}(t)\right) \otimes I_{N+1}} \\
& \text { and } \\
& {\left[\sin ^{2}(t\langle H\rangle) \sin ^{2}\left(t\left\langle H^{\prime}\right\rangle\right)+\cos ^{2}(t\langle H\rangle) \cos ^{2}\left(t\left\langle H^{\prime}\right\rangle\right)\right] t^{2}\left(\sigma_{m}^{2}+\sigma_{V}^{2}(t)+\sigma_{m}^{\prime 2}+\sigma_{V}^{\prime 2}(t)\right) \otimes I_{N+1}(30)}
\end{aligned}
$$

Now we use Portmanteau's theorem [1] on Equation (28) with respect to the random variable $\mathfrak{I}=H+H^{\prime}$, express the appearing distribution densities in terms of the phase space variables and integrate with respect to infinitesimal volumes $V_{\text {inf }}$ to obtain the relation

$$
\begin{aligned}
& \left(\prod_{n=1}^{N}\left[\int \mathrm{d} r_{n}\right] \prod_{n=1}^{N+1}\left[\int \frac{\mathrm{d} p_{n}}{2 \pi}\right] \prod_{n=1}^{N}\left[\int \mathrm{d} r_{n}^{\prime}\right] \prod_{n=1}^{N+1}\left[\int \frac{\mathrm{d} p_{n}^{\prime}}{2 \pi}\right]\right)_{V_{\mathrm{inf}}} \\
& \times\left\{\exp \left[-i \varepsilon \sum_{n=1}^{N+1}\left[\left(H\left(p_{n}, r_{n}\right)+H^{\prime}\left(p_{n}^{\prime}, r_{n}^{\prime}\right)\right)\right]\right]-\exp \left[-i t\left(\langle H(t)\rangle+\left\langle H^{\prime}(t)\right\rangle\right)\right]\right.
\end{aligned}
$$




$$
\left.-\frac{1}{\sqrt{N+1}}\left\{\prod_{n=1}^{N+1}\left[f_{1}\left(p_{n}, r_{n}\right) f_{1}^{\prime}\left(p_{n}^{\prime}, r_{n}^{\prime}\right)\right]-i \prod_{n=1}^{N+1}\left[g_{1}\left(p_{n}, r_{n}\right) g_{1}^{\prime}\left(p_{n}^{\prime}, r_{n}^{\prime}\right)\right]\right\}\right\}=0
$$

Since the covariance matrices are diagonal we have set the Gaussian distributions of the random variables $Y$ and $W$, as products of the functions

$$
\begin{aligned}
& f_{1}\left(p_{n}, r_{n}\right)=\frac{1}{2 \pi \sigma_{m} \sigma_{V}(t) t^{2}\left[\sin ^{2}(t\langle H\rangle) \cos ^{2}\left(t\left\langle H^{\prime}\right\rangle\right)+\cos ^{2}(t\langle H\rangle) \sin ^{2}\left(t\left\langle H^{\prime}\right\rangle\right)\right]} \\
& \times \exp \left\{-\frac{1}{t^{2}\left[\sin ^{2}(t\langle H\rangle) \cos ^{2}\left(t\left\langle H^{\prime}\right\rangle\right)+\cos ^{2}(t\langle H\rangle) \sin ^{2}\left(t\left\langle H^{\prime}\right\rangle\right)\right]}\left[\frac{p_{n}^{2}}{2 \sigma_{m}^{2}}+\frac{r_{n}^{2}}{2 \sigma_{V}^{2}(t)}\right]\right\}
\end{aligned}
$$

and

$$
\begin{aligned}
& g_{1}\left(p_{n}, r_{n}\right)=\frac{1}{2 \pi \sigma_{m} \sigma_{V}(t) t^{2}\left[\sin ^{2}(t\langle H\rangle) \sin ^{2}\left(t\left\langle H^{\prime}\right\rangle\right)+\cos ^{2}(t\langle H\rangle) \cos ^{2}\left(t\left\langle H^{\prime}\right\rangle\right)\right]} \\
& \times \exp \left\{-\frac{1}{t^{2}\left[\sin ^{2}(t\langle H\rangle) \sin ^{2}\left(t\left\langle H^{\prime}\right\rangle\right)+\cos ^{2}(t\langle H\rangle) \cos ^{2}\left(t\left\langle H^{\prime}\right\rangle\right)\right]}\left[\frac{p_{n}^{2}}{2 \sigma_{m}^{2}}+\frac{r_{n}^{2}}{2 \sigma_{V}^{2}(t)}\right]\right\}
\end{aligned}
$$

In the case of the primed factors in Equation (31) we use the same expressions with primed variances.

Now in the integral of Equation (31) we apply the mean value theorem and multiply the result with the following function of phase space variables:

$$
\begin{aligned}
& h\left(r_{0 i}, r_{01}, r_{02}, \cdots, r_{0 N}, r_{0 f} ; r_{0 i}^{\prime}, r_{01}^{\prime}, r_{02}^{\prime}, \cdots, r_{0 N}^{\prime}, r_{0 f}^{\prime}\right) \\
& \times \exp \left\{i \sum_{n=1}^{N+1}\left[p_{0 n}\left(r_{0 n}-r_{0 n-1}\right)+p_{0 n}^{\prime}\left(r_{0 n}^{\prime}-r_{0 n-1}^{\prime}\right)-\varepsilon H_{I}\left(r_{0 n}, r_{0 n}^{\prime}\right)\right]\right\}
\end{aligned}
$$

where the point

$$
\left(p_{01}, p_{02}, \cdots, p_{0 N}, p_{0 N+1}, r_{01}, r_{02}, \cdots, r_{0 N}, p_{01}^{\prime}, p_{02}^{\prime}, \cdots, p_{0 N}^{\prime}, p_{0 N+1}^{\prime}, r_{01}^{\prime}, r_{02}^{\prime}, \cdots, r_{0 N}^{\prime}\right)
$$

of index 0 is the same point appearing in the mean value theorem. Then we sum over all the infinitesimal volumes partitioning the integration space and let them shrink to points to eventually obtain the form (see Equations (5), (6))

$$
\begin{aligned}
& K^{(N)}\left(r_{f}, r_{f}^{\prime} ; r_{i}, r_{i}^{\prime} ; t\right) \\
& =\prod_{n=1}^{N}\left[\int \mathrm{d} r_{n}\right] \prod_{n=1}^{N+1}\left[\int \frac{\mathrm{d} p_{n}}{2 \pi}\right] \prod_{n=1}^{N}\left[\int \mathrm{d} r_{n}^{\prime}\right] \prod_{n=1}^{N+1}\left[\int \frac{\mathrm{d} p_{n}^{\prime}}{2 \pi}\right] h\left(r_{i}, r_{1}, r_{2}, \cdots, r_{N}, r_{f} ; r_{i}^{\prime}, r_{1}^{\prime}, r_{2}^{\prime}, \cdots, r_{N}^{\prime}, r_{f}^{\prime}\right) \\
& \times \exp \left\{i \sum_{n=1}^{N+1}\left[p_{n}\left(r_{n}-r_{n-1}\right)+p_{n}^{\prime}\left(r_{n}^{\prime}-r_{n-1}^{\prime}\right)-\varepsilon H_{I}\left(r_{n}, r_{n}^{\prime}\right)\right]\right\} \exp \left(-i t\left(\langle H(t)\rangle+\left\langle H^{\prime}(t)\right\rangle\right)\right) \\
& +\frac{1}{\sqrt{N+1}} \prod_{n=1}^{N}\left[\int \mathrm{d} r_{n}\right] \prod_{n=1}^{N+1}\left[\int \frac{\mathrm{d} p_{n}}{2 \pi}\right] \prod_{n=1}^{N}\left[\int \mathrm{d} r_{n}^{\prime}\right] \prod_{n=1}^{N+1}\left[\int \frac{\mathrm{d} p_{n}^{\prime}}{2 \pi}\right] \\
& \times h\left(r_{i}, r_{1}, r_{2}, \cdots, r_{N}, r_{f} ; r_{i}^{\prime}, r_{1}^{\prime}, r_{2}^{\prime}, \cdots, r_{N}^{\prime}, r_{f}^{\prime}\right) \exp \left\{i \sum_{n=1}^{N+1}\left[p_{n}\left(r_{n}-r_{n-1}\right)+p_{n}^{\prime}\left(r_{n}^{\prime}-r_{n-1}^{\prime}\right)-\varepsilon H_{I}\left(r_{n}, r_{n}^{\prime}\right)\right]\right\} \\
& \times\left\{\prod_{n=1}^{N+1}\left[f_{1}\left(p_{n}, r_{n}\right) f_{1}^{\prime}\left(p_{n}^{\prime}, r_{n}^{\prime}\right)\right]-i \prod_{n=1}^{N+1}\left[g_{1}\left(p_{n}, r_{n}\right) g_{1}^{\prime}\left(p_{n}^{\prime}, r_{n}^{\prime}\right)\right]\right\}
\end{aligned}
$$

Further on performing the integrations on all the $p_{n}, p_{n}^{\prime}, r_{n}, r_{n}^{\prime}$ variables in the first term in Equation (35) and on the $p_{n}, p_{n}^{\prime}$ variables in the second 
one, we obtain the theorem of the solution of the sign problem for Equation (4), according to which as $N \rightarrow \infty$

$$
\begin{aligned}
& K^{(N)}\left(r_{f}, r_{f}^{\prime} ; r_{i}, r_{i}^{\prime} ; t\right) \\
& \cong \delta\left(r_{f}-r_{i}\right) \delta\left(r_{f}^{\prime}-r_{i}^{\prime}\right) h\left(r_{f}, r_{f}, r_{f}, \cdots, r_{f}, r_{f}, r_{f}^{\prime}, r_{f}^{\prime}, r_{f}^{\prime}, \cdots, r_{f}^{\prime}, r_{f}^{\prime}\right) \\
& \times \exp \left(-i t H_{I}\left(r_{f}, r_{f}^{\prime}\right)\right) \exp \left(-i t\left(\langle H(t)\rangle+\left\langle H^{\prime}(t)\right\rangle\right)\right) \\
& +\frac{1}{\sqrt{N+1}} \prod_{n=1}^{N}\left[\int \mathrm{d} r_{n}\right] \prod_{n=1}^{N}\left[\int \mathrm{d} r_{n}^{\prime}\right] h\left(r_{i}, r_{1}, r_{2}, \cdots, r_{N}, r_{f} ; r_{i}^{\prime}, r_{1}^{\prime}, r_{2}^{\prime}, \cdots, r_{N}^{\prime}, r_{f}^{\prime}\right) \\
& \quad \times \exp \left\{-i \varepsilon \sum_{n=1}^{N+1} H_{I}\left(r_{n}, r_{n}^{\prime}\right)\right\}\left\{\prod_{n=1}^{N+1}\left[f_{2}\left(r_{n}, r_{n-1}\right) f_{2}^{\prime}\left(r_{n}^{\prime}, r_{n-1}^{\prime}\right)\right]\right. \\
& \left.-i \prod_{n=1}^{N+1}\left[g_{2}\left(r_{n}, r_{n-1}\right) g_{2}^{\prime}\left(r_{n}^{\prime}, r_{n-1}^{\prime}\right)\right]\right\}
\end{aligned}
$$

We have used the functions

$$
\begin{aligned}
f_{2}\left(r_{n}, r_{n-1}\right) & \frac{1}{2 \pi \sqrt{2 \pi} \sigma_{V}(t) t \sqrt{\left[\sin ^{2}(t\langle H\rangle) \cos ^{2}\left(t\left\langle H^{\prime}\right\rangle\right)+\cos ^{2}(t\langle H\rangle) \sin ^{2}\left(t\left\langle H^{\prime}\right\rangle\right)\right]}} \\
& \times \exp \left\{-\frac{1}{2} \sigma_{m}^{2} t^{2}\left[\sin ^{2}(t\langle H\rangle) \cos ^{2}\left(t\left\langle H^{\prime}\right\rangle\right)+\cos ^{2}(t\langle H\rangle) \sin ^{2}\left(t\left\langle H^{\prime}\right\rangle\right)\right]\left(r_{n}-r_{n-1}\right)^{2}\right. \\
& \left.-\frac{r_{n}^{2}}{2 \sigma_{V}^{2}(t) t^{2}\left[\sin ^{2}(t\langle H\rangle) \cos ^{2}\left(t\left\langle H^{\prime}\right\rangle\right)+\cos ^{2}(t\langle H\rangle) \sin ^{2}\left(t\left\langle H^{\prime}\right\rangle\right)\right]}\right\} \\
g_{2}\left(r_{n}, r_{n-1}\right) & \frac{1}{2 \pi \sqrt{2 \pi} \sigma_{V}(t) t \sqrt{\left[\sin ^{2}(t\langle H\rangle) \sin ^{2}\left(t\left\langle H^{\prime}\right\rangle\right)+\cos ^{2}(t\langle H\rangle) \cos ^{2}\left(t\left\langle H^{\prime}\right\rangle\right)\right]}} \\
& \times \exp \left\{-\frac{1}{2} \sigma_{m}^{2} t^{2}\left[\sin ^{2}(t\langle H\rangle) \sin ^{2}\left(t\left\langle H^{\prime}\right\rangle\right)+\cos ^{2}(t\langle H\rangle) \cos ^{2}\left(t\left\langle H^{\prime}\right\rangle\right)\right]\left(r_{n}-r_{n-1}\right)^{2}\right. \\
& -\frac{r_{n}^{2}}{\left.2 \sigma_{V}^{2}(t) t^{2}\left[\sin ^{2}(t\langle H\rangle) \sin ^{2}\left(t\left\langle H^{\prime}\right\rangle\right)+\cos ^{2}(t\langle H\rangle) \cos ^{2}\left(t\left\langle H^{\prime}\right\rangle\right)\right]\right\}}
\end{aligned}
$$

In the case of the primed functions in Equation (36) we use the same expressions with primed variances.

Now we state that under appropriate conditions only the first term involving the delta functions in Equation (36) contribute to the exact result as $N \rightarrow \infty$. In that case, we call that term sign solved propagator (SSP). To prove that, we diagonalize and integrate the Gaussian products $\prod_{n=1}^{N+1}\left[f_{2}\left(r_{n}, r_{n-1}\right) f_{2}^{\prime}\left(r_{n}^{\prime}, r_{n-1}^{\prime}\right)\right]$ and $\prod_{n=1}^{N+1}\left[g_{2}\left(r_{n}, r_{n-1}\right) g_{2}^{\prime}\left(r_{n}^{\prime}, r_{n-1}^{\prime}\right)\right]$ via the same procedure as in ref. [5] and assume the condition

$$
\left|h\left(r_{i}, r_{1}, r_{2}, \cdots, r_{N}, r_{f} ; r_{i}^{\prime}, r_{1}^{\prime}, r_{2}^{\prime}, \cdots, r_{N}^{\prime}, r_{f}^{\prime}\right)\right| \leq b(N+1)^{\gamma} C^{2 N+4}, \quad b, C>0, \gamma \in R
$$

Then if $\mathfrak{R}$ is the second term on right-hand side of (36) and the range of the 
$r_{n}$ variables is from 0 to $\infty$ we obtain the following upper bound of the $|\Re|$

$$
\begin{aligned}
|\mathfrak{R}| \leq & b_{1}(2 \pi)^{3} \sigma_{V}(t) \sigma_{V}^{\prime}(t) t^{2}\left[\sin ^{2}(t\langle H\rangle) \cos ^{2}\left(t\left\langle H^{\prime}\right\rangle\right)+\cos ^{2}(t\langle H\rangle) \sin ^{2}\left(t\left\langle H^{\prime}\right\rangle\right)\right] \\
& \times\left\{\frac{(N+1)^{\gamma}}{\sqrt{N+1}} \prod_{n=0}^{N+1}\left[\frac{C}{4 \pi \sqrt{\Lambda_{s n}^{(N+2)}}} \frac{C}{4 \pi \sqrt{\Lambda_{s n}^{(N+2)}}}\right]\right\} \\
& +b_{2}(2 \pi)^{3} \sigma_{V}(t) \sigma_{V}^{\prime}(t) t^{2}\left[\sin ^{2}(t\langle H\rangle) \sin ^{2}\left(t\left\langle H^{\prime}\right\rangle\right)+\cos ^{2}(t\langle H\rangle) \cos ^{2}\left(t\left\langle H^{\prime}\right\rangle\right)\right] \\
& \times\left\{\frac{(N+1)^{\gamma}}{\sqrt{N+1}} \prod_{n=0}^{N+1}\left[\frac{C}{4 \pi \sqrt{\Lambda_{c n}^{(N+2)}}} \frac{C}{4 \pi \sqrt{\Lambda_{c n}^{(N+2)}}}\right]\right\}
\end{aligned}
$$

where

$$
\begin{aligned}
\Lambda_{s n}^{(N+2)}= & 1+2\left(1+\xi_{s N+2}^{(n)}\right) \sigma_{m}^{2} \sigma_{V}^{2}(t) t^{4}\left[\sin ^{2}(t\langle H\rangle) \cos ^{2}\left(t\left\langle H^{\prime}\right\rangle\right)\right. \\
& \left.+\cos ^{2}(t\langle H\rangle) \sin ^{2}\left(t\left\langle H^{\prime}\right\rangle\right)\right]^{2} \\
\Lambda_{c n}^{(N+2)}= & 1+2\left(1+\xi_{c N+2}^{(n)}\right) \sigma_{m}^{2} \sigma_{V}^{2}(t) t^{4}\left[\sin ^{2}(t\langle H\rangle) \sin ^{2}\left(t\left\langle H^{\prime}\right\rangle\right)\right. \\
& \left.+\cos ^{2}(t\langle H\rangle) \cos ^{2}\left(t\left\langle H^{\prime}\right\rangle\right)\right]^{2}
\end{aligned}
$$

Again in the case of the primed $\Lambda_{s n}^{\prime(N+2)}, \Lambda_{c n}^{\prime(N+2)}$ we use the same expressions with primed variances. $\xi_{s N+2}^{(n)}, \quad \xi_{c N+2}^{(n)}$ are constants such that $\xi_{\{s\}_{N+2}^{(n)}}^{(c)}>-1$.

Therefore if the expressions in the curly brackets in Equation (40) tend to zero as $N \rightarrow \infty$, then the first term in Equation (36) is exact as $N \rightarrow \infty$ and corresponds to the sign solved propagator. This is the case if $C<4 \pi$, since $\Lambda_{\left\{\begin{array}{l}s \\ c\end{array}\right\}^{n}}^{(N+2)} \geq 1, \quad \Lambda_{\left\{\begin{array}{l}(N+2) \\ c\end{array}\right\}^{n}}^{(N+2)} \geq 1 \quad$ (see Equations (41), (42)). Concluding we have proven the sign solved propagator theorem according to which

$$
\begin{aligned}
& K\left(r_{f}, r_{f}^{\prime} ; r_{i}, r_{i}^{\prime} ; t\right) \\
& =\delta\left(r_{f}-r_{i}\right) \delta\left(r_{f}^{\prime}-r_{i}^{\prime}\right) \lim _{N \rightarrow \infty} h(\underbrace{r_{f}, r_{f}, r_{f}, \cdots, r_{f}, r_{f}}_{N+2}, \underbrace{r_{f}^{\prime}, r_{f}^{\prime}, r_{f}^{\prime}, \cdots, r_{f}^{\prime}, r_{f}^{\prime}}_{N+2}) \\
& \times \exp \left(-i t H_{I}\left(r_{f}, r_{f}^{\prime}\right)\right) \exp \left(-i t\left(\langle H(t)\rangle+\left\langle H^{\prime}(t)\right\rangle\right)\right)
\end{aligned}
$$

\section{The 3D Helium Path Integral}

We consider the 3D Helium [9] [10] [11] [12]. Its Hamiltonian has the form

$$
H_{H e}^{3 D}=\frac{\vec{p}_{1}^{2}}{2}+\frac{\vec{p}_{2}^{2}}{2}-\frac{Z}{\left|\vec{r}_{1}\right|}-\frac{Z}{\left|\vec{r}_{2}\right|}+\frac{1}{\left|\vec{r}_{1}-\vec{r}_{2}\right|}
$$

where $Z$ is the atomic number. $\vec{r}_{1}$ and $\vec{r}_{2}$ are the position vectors of the two electrons with respect to the nucleus.

The path integral of a Helium atom has the form

$$
K_{1}\left(\vec{r}_{1 f}, \vec{r}_{2 f}, t_{f} ; \vec{r}_{1 i}, \vec{r}_{2 i}, t_{i}\right)=\lim _{N \rightarrow \infty} \prod_{n=1}^{N} \int_{-\infty}^{\infty} \mathrm{d} \vec{r}_{1 n} \prod_{n=1}^{N+1}\left[\int_{-\infty}^{\infty} \frac{\mathrm{d} \vec{p}_{1 n}}{(2 \pi)^{3}}\right] \prod_{n=1}^{N} \int_{-\infty}^{\infty} \mathrm{d} \vec{r}_{2 n} \prod_{n=1}^{N+1}\left[\int_{-\infty}^{\infty} \frac{\mathrm{d} \vec{p}_{2 n}}{(2 \pi)^{3}}\right]
$$




$$
\begin{aligned}
& \times \exp \left\{i \sum _ { n = 1 } ^ { N + 1 } \left[\vec{p}_{1 n} \cdot\left(\vec{r}_{1 n}-\vec{r}_{1 n-1}\right)+\vec{p}_{2 n} \cdot\left(\vec{r}_{2 n}-\vec{r}_{2 n-1}\right)\right.\right. \\
& \left.\left.-\varepsilon\left(\frac{\vec{p}_{1 n}^{2}}{2}+\frac{\vec{p}_{2 n}^{2}}{2}-\frac{Z}{\left|\vec{r}_{1 n}\right|}-\frac{Z}{\left|\vec{r}_{2 n}\right|}+\frac{1}{\left|\vec{r}_{1 n}-\vec{r}_{2 n}\right|}\right)\right]\right\}
\end{aligned}
$$

where $\varepsilon=\frac{t_{f}-t_{i}}{N+1}$. We have set $\vec{r}_{10}=\vec{r}_{1 i}, \vec{r}_{20}=\vec{r}_{2 i}, \vec{r}_{1 N+1}=\vec{r}_{1 f}$ and $\vec{r}_{2 N+1}=\vec{r}_{2 f}$. Now we observe that we can write

$$
\begin{aligned}
\frac{1}{\left|\vec{r}_{1 n}-\vec{r}_{2 n}\right|} & =\frac{1}{\sqrt{r_{1 n}^{2}+r_{2 n}^{2}-2 \vec{r}_{1 n} \cdot \vec{r}_{2 n}}}=\frac{1}{\sqrt{r_{1 n}^{2}+r_{2 n}^{2}-2 r_{1 n} r_{2 n} \cos \vartheta_{12 n}}} \\
& =\frac{1}{\sqrt{r_{1 n}^{2}+r_{2 n}^{2}}} \frac{1}{\sqrt{1-\frac{2 r_{1 n} r_{2 n}}{r_{1 n}^{2}+r_{2 n}^{2}} \cos \vartheta_{12 n}}}=\frac{1}{\sqrt{r_{1 n}^{2}+r_{2 n}^{2}}} \frac{1}{\sqrt{1-\frac{2}{\frac{r_{1 n}}{r_{2 n}}+\frac{r_{2 n}}{r_{1 n}}} \cos \vartheta_{12 n}}} \\
& =\frac{1}{\sqrt{r_{1 n}^{2}+r_{2 n}^{2}}} \frac{1}{\sqrt{1-\alpha\left(r_{1 n}, r_{2 n}\right) \cos \vartheta_{12 n}}}
\end{aligned}
$$

where

$$
\alpha\left(r_{1 n}, r_{2 n}\right)=\frac{2}{\frac{r_{1 n}}{r_{2 n}}+\frac{r_{2 n}}{r_{1 n}}} \leq 1
$$

and $r_{1 n}=\left|\vec{r}_{1 n}\right|, \quad r_{2 n}=\left|\vec{r}_{2 n}\right|$. Therefore the path integral expression (45) becomes

$$
\begin{aligned}
& K_{1}\left(\vec{r}_{1 f}, \vec{r}_{2 f}, t_{f} ; \vec{r}_{1 i}, \vec{r}_{2 i}, t_{i}\right) \\
& =\lim _{N \rightarrow \infty} \prod_{n=1}^{N} \int_{-\infty}^{\infty} \mathrm{d} \vec{r}_{1 n} \prod_{n=1}^{N+1}\left[\int_{-\infty}^{\infty} \frac{\mathrm{d} \vec{p}_{1 n}}{(2 \pi)^{3}}\right] \prod_{n=1}^{N} \int_{-\infty}^{\infty} \mathrm{d} \vec{r}_{2 n} \prod_{n=1}^{N+1}\left[\int_{-\infty}^{\infty} \frac{\mathrm{d} \vec{p}_{2 n}}{(2 \pi)^{3}}\right] \\
& \times \exp \left\{i \sum _ { n = 1 } ^ { N + 1 } \left[\vec{p}_{1 n} \cdot\left(\vec{r}_{1 n}-\vec{r}_{1 n-1}\right)+\vec{p}_{2 n} \cdot\left(\vec{r}_{2 n}-\vec{r}_{2 n-1}\right)\right.\right. \\
& \left.\left.-\varepsilon\left(\frac{\vec{p}_{1 n}^{2}}{2}+\frac{\vec{p}_{2 n}^{2}}{2}-\frac{Z}{r_{1 n}}-\frac{Z}{r_{2 n}}+\frac{1}{\sqrt{r_{1 n}^{2}+r_{2 n}^{2}}} \frac{1}{\sqrt{1-\alpha\left(r_{1 n}, r_{2 n}\right) \cos \vartheta_{12 n}}}\right)\right]\right\}
\end{aligned}
$$

At this point, we insert in Equation (48) a delta function to get the form

$$
\begin{aligned}
K_{1}\left(\vec{r}_{1 f}, \vec{r}_{2 f}, t_{f} ; \vec{r}_{1 i}, \vec{r}_{2 i}, t_{i}\right)= & \lim _{N \rightarrow \infty} \prod_{n=1}^{N} \int_{-\infty}^{\infty} \mathrm{d} \vec{r}_{1 n} \prod_{n=1}^{N+1}\left[\int_{-\infty}^{\infty} \frac{\mathrm{d} \vec{p}_{1 n}}{(2 \pi)^{3}}\right] \prod_{n=1}^{N} \int_{-\infty}^{\infty} \mathrm{d} \vec{r}_{2 n} \prod_{n=1}^{N+1}\left[\int_{-\infty}^{\infty} \frac{\mathrm{d} \vec{p}_{2 n}}{(2 \pi)^{3}}\right] \\
& \times \prod_{n=1}^{N+1}\left[\int_{-\infty}^{\infty} \mathrm{d} u_{n}\right] \prod_{n=1}^{N+1}\left[\delta\left(u_{n}-\alpha\left(r_{1 n}, r_{2 n}\right) \cos \vartheta_{12 n}\right)\right] \\
& \times \exp \left\{i \sum _ { n = 1 } ^ { N + 1 } \left[\vec{p}_{1 n} \cdot\left(\vec{r}_{1 n}-\vec{r}_{1 n-1}\right)+\vec{p}_{2 n} \cdot\left(\vec{r}_{2 n}-\vec{r}_{2 n-1}\right)\right.\right. \\
& \left.\left.-\varepsilon\left(\frac{\vec{p}_{1 n}^{2}}{2}+\frac{\vec{p}_{2 n}^{2}}{2}-\frac{Z}{r_{1 n}}-\frac{Z}{r_{2 n}}+\frac{1}{\sqrt{r_{1 n}^{2}+r_{2 n}^{2}}} \frac{1}{\sqrt{1-u_{n}}}\right)\right]\right\}
\end{aligned}
$$


Further, the delta functions in Equation (49) have the representation

$$
\begin{aligned}
\delta\left(u_{n}-\alpha_{n} \cos \vartheta_{12 n}\right) & =\frac{1}{2 \pi} \int_{-\infty}^{\infty} \mathrm{d} \lambda_{n} \mathrm{e}^{-i \lambda_{n} u_{n}} \mathrm{e}^{i \lambda_{n} \alpha_{n} \cos \vartheta_{2 n}} \\
& =\frac{1}{2 \pi} \sum_{l_{n}=0}^{\infty}\left(2 l_{n}+1\right) i^{l_{n}} P_{l_{n}}\left(\cos \vartheta_{12 n}\right) \int_{-\infty}^{\infty} \mathrm{d} \lambda_{n} \mathrm{e}^{-i \lambda_{n} u_{n}} j_{l_{n}}\left(\alpha_{n} \lambda_{n}\right)
\end{aligned}
$$

where $\alpha_{n}=\alpha\left(r_{1 n}, r_{2 n}\right)$. We set the integrals appearing in Equation (50) as

$$
\frac{1}{2 \pi} \int_{-\infty}^{\infty} \mathrm{d} \lambda \mathrm{e}^{-i \lambda u} j_{l}(\alpha \lambda)=g_{l}(u)
$$

Then after standard calculations, we obtain the results

$$
g_{0}(u)= \begin{cases}\frac{1}{2 \alpha} & \text { if }|u|<\alpha \\ 0 & \text { otherwise }\end{cases}
$$

and

$$
g_{1}(u)= \begin{cases}-\frac{i u}{2 \alpha^{2}} & \text { if }|u|<\alpha \\ 0 & \text { otherwise }\end{cases}
$$

Moreover the following recurrence relation is valid

$$
g_{l+1}(u)=\frac{l}{l+1} g_{l-1}(u)-i \frac{2 l+1}{l+1} \frac{u}{\alpha} g_{l}(u)
$$

Therefore we can perform the integrations in Equation (50) according to Equations (51)-(54) and place the results in Equation (49) after keeping leading terms with respect the $l_{n}$. After the angular decomposition, the angular part of higher-order terms in the expansion (50) contributes infinities as $N \rightarrow \infty$ and we drop them (see below). Now expression (49) becomes

$$
\begin{aligned}
& K_{1}\left(\vec{r}_{1 f}, \vec{r}_{2 f}, t_{f} ; \vec{r}_{1 i}, \vec{r}_{2 i}, t_{i}\right) \\
& =\lim _{N \rightarrow \infty} \prod_{n=1}^{N} \int_{-\infty}^{\infty} \mathrm{d} \vec{r}_{1 n} \prod_{n=1}^{N+1}\left[\int_{-\infty}^{\infty} \frac{\mathrm{d} \vec{p}_{1 n}}{(2 \pi)^{3}}\right] \prod_{n=1}^{N} \int_{-\infty}^{\infty} \mathrm{d} \vec{r}_{2 n} \prod_{n=1}^{N+1}\left[\int_{-\infty}^{\infty} \frac{\mathrm{d} \vec{p}_{2 n}}{(2 \pi)^{3}} \prod_{n=1}^{N+1}\left[\int_{-\alpha_{n}}^{\alpha_{n}} \mathrm{~d} u_{n}\right]\right. \\
& \times \prod_{n=1}^{N}\left[g_{0}\left(u_{n}\right)\right]\left(\sum_{l_{N+1}=0}^{\infty}\left(2 l_{N+1}+1\right) i^{l_{N+1}} P_{l_{N+1}}\left(\cos \vartheta_{12 N+1}\right) g_{l_{N+1}}\left(u_{N+1}\right)\right) \\
& \times \exp \left\{i \sum _ { n = 1 } ^ { N + 1 } \left[\vec{p}_{1 n} \cdot\left(\vec{r}_{1 n}-\vec{r}_{1 n-1}\right)+\vec{p}_{2 n} \cdot\left(\vec{r}_{2 n}-\vec{r}_{2 n-1}\right)\right.\right. \\
& \left.\left.-\varepsilon\left(\frac{\vec{p}_{1 n}^{2}}{2}+\frac{\vec{p}_{2 n}^{2}}{2}-\frac{Z}{r_{1 n}}-\frac{Z}{r_{2 n}}+\frac{1}{\sqrt{r_{1 n}^{2}+r_{2 n}^{2}}} \frac{1}{\sqrt{1-u_{n}}}\right)\right]\right\}
\end{aligned}
$$

We have set the range of integration over $u_{n}$ within the interval from $-\alpha_{n}$ to $\alpha_{n}$ as otherwise, the functions $g_{l}(u)$ are zero (see Equations (51)-(54)). Moreover in Equation (55) in the case of the $N+1$ factor, we have kept the full series appearing in Equation (50) as it involves the final coordinates.

Now in Equation (55) we perform certain standard manipulations including 
angular decomposition of the path integral and the use of the addition theorem of spherical harmonics for the Legendre polynomial $P_{l_{N+1}}\left(\cos \vartheta_{12 N+1}\right)$ to obtain the result

$$
\begin{aligned}
& K_{1}\left(\vec{r}_{1 f}, \vec{r}_{2 f}, t_{f} ; \vec{r}_{1 i}, \vec{r}_{2 i}, t_{i}\right) \\
& \left.=\frac{4 \pi}{r_{1 f} r_{1 i} r_{2 f} r_{2 i}} \sum_{l=0}^{\infty} \sum_{p=-l}^{l} \sum_{q_{1}=0}^{\infty} \sum_{m_{1}=-q_{1}}^{q_{1}} \sum_{q_{2}=0}^{\infty} \sum_{m_{2}=-q_{2}}^{q_{2}} K_{l q_{1} q_{2}}\left(r_{1 f}, r_{2 f}, t_{f} ; r_{1 i}, r_{2 i}, t_{i}\right)\right)^{l} Y_{q_{1} m_{1}}\left(\vartheta_{1 f}, \varphi_{1 f}\right) \\
& \times Y_{l p}\left(\vartheta_{1 f}, \varphi_{1 f}\right) Y_{q_{2} m_{2}}\left(\vartheta_{2 f}, \varphi_{2 f}\right) Y_{l p}^{*}\left(\vartheta_{2 f}, \varphi_{2 f}\right) Y_{q_{1} m_{1}}^{*}\left(\vartheta_{1 i}, \varphi_{1 i}\right) Y_{q_{2} m_{2}}^{*}\left(\vartheta_{2 i}, \varphi_{2 i}\right)
\end{aligned}
$$

The term $K_{l q_{1} q_{2}}\left(r_{1 f}, r_{2 f}, t_{f} ; r_{1 i}, r_{2 i}, t_{i}\right)$ corresponds to the path integral

$$
\begin{aligned}
& K_{l q_{1} q_{2}}\left(r_{1 f}, r_{2 f}, t_{f} ; r_{1 i}, r_{2 i}, t_{i}\right) \\
& =\lim _{N \rightarrow \infty} \prod_{n=1}^{N} \int_{0}^{\infty} \mathrm{d} r_{1 n} \prod_{n=1}^{N+1}\left[\int_{-\infty}^{\infty} \frac{\mathrm{d} p_{1 n}}{2 \pi}\right] \prod_{n=1}^{N} \int_{0}^{\infty} \mathrm{d} r_{2 n} \prod_{n=1}^{N+1}\left[\int_{-\infty}^{\infty} \frac{\mathrm{d} p_{2 n}}{2 \pi}\right] \\
& \times \exp \left\{i \sum _ { n = 1 } ^ { N + 1 } \left[p_{1 n}\left(r_{1 n}-r_{1 n-1}\right)+p_{2 n}\left(r_{2 n}-r_{2 n-1}\right)-\varepsilon\left(\frac{p_{1 n}^{2}}{2}+\frac{q_{1}\left(q_{1}+1\right)}{2 r_{1 n}^{2}}-\frac{Z}{r_{1 n}}\right.\right.\right. \\
& \left.\left.\left.+\frac{p_{2 n}^{2}}{2}+\frac{q_{2}\left(q_{2}+1\right)}{2 r_{2 n}^{2}}-\frac{Z}{r_{2 n}}\right)\right]\right\} G_{l}\left(r_{11}, r_{12}, \cdots, r_{1 N+1}, r_{21}, r_{22}, \cdots, r_{2 N+1}\right)
\end{aligned}
$$

We have set $r_{10}=r_{1 i}, r_{20}=r_{2 i}, r_{1 N+1}=r_{1 f}$ and $r_{2 N+1}=r_{2 f}$ while the factor $G_{l}\left(r_{11}, r_{12}, \cdots, r_{1 N+1}, r_{21}, r_{22}, \cdots, r_{2 N+1}\right)$ appearing above has the form

$$
\begin{aligned}
& G_{l}\left(r_{11}, r_{12}, \cdots, r_{1 N+1}, r_{21}, r_{22}, \cdots, r_{2 N+1}\right) \\
& =\prod_{n=1}^{N+1}\left[\int_{-\alpha_{n}}^{\alpha_{n}} \mathrm{~d} u_{n}\right] \prod_{n=1}^{N}\left[g_{0}\left(u_{n}\right)\right] g_{l}\left(u_{N+1}\right) \exp \left\{-i \varepsilon \sum_{n=1}^{N+1}\left[\frac{1}{\sqrt{r_{1 n}^{2}+r_{2 n}^{2}}} \frac{1}{\sqrt{1-u_{n}}}\right]\right\}
\end{aligned}
$$

Some of the integrals in Equation (58) are given in Appendix A.

According to our methods in the case of path integrals which involve inner or outer products in the interaction term or the potential, after the angular decomposition, we evaluate the infinite product from $n=1$ to $n=N$ as $N \rightarrow \infty$ and integrate the $n=N+1$ factor considering the parameter $\varepsilon$ as a one form. So the infinite product becomes

$$
\lim _{N \rightarrow \infty} \prod_{n=1}^{N}\left[\int_{-\alpha_{n}}^{\alpha_{n}} \frac{\mathrm{d} u_{n}}{2 \alpha_{n}}\right] \exp \left\{-i \varepsilon \sum_{n=1}^{N+1}\left[\frac{1}{\sqrt{r_{1 n}^{2}+r_{2 n}^{2}}} \frac{1}{\sqrt{1-u_{n}}}\right]\right\}=\exp \left[-i \int_{t_{i}}^{t_{\bar{f}}} \mathrm{~d} \tau \frac{1}{r_{>}(\tau)}\right]
$$

where $r_{>}=\max \left(r_{1}, r_{2}\right)$ and $r_{<}=\min \left(r_{1}, r_{2}\right)$.

That result is the same with an average of the interaction term over the solid angle. So there remains only the zero-order term in a relevant expansion over spherical harmonics.

Now we combine Equations (57)-(59) to get (see Equation (A5) in Appendix A as well)

$$
\begin{aligned}
& K_{l q_{1} q_{2}}\left(r_{1 f}, r_{2 f}, t_{f} ; r_{1 i}, r_{2 i}, t_{i}\right) \\
& =J_{l}\left(\frac{t_{f}-t_{i}}{\sqrt{r_{1 f}^{2}+r_{2 f}^{2}}}, \alpha_{f} \int_{0}^{\infty} D r_{1} \int_{-\infty}^{\infty} \frac{D p_{1}}{2 \pi} \int_{0}^{\infty} D r_{2} \int_{-\infty}^{\infty} \frac{D p_{2}}{2 \pi}\right.
\end{aligned}
$$




$$
\begin{aligned}
& \times \exp \left\{i \int _ { t _ { i } } ^ { t _ { f } } \mathrm { d } \tau \left[p_{1} \dot{r}_{1}+p_{2} \dot{r}_{2}-\left(\frac{p_{1}^{2}}{2}+\frac{q_{1}\left(q_{1}+1\right)}{2 r_{1}^{2}}-\frac{Z}{r_{1}}\right.\right.\right. \\
& \left.\left.\left.+\frac{p_{2}^{2}}{2}+\frac{q_{2}\left(q_{2}+1\right)}{2 r_{2}^{2}}-\frac{Z}{r_{2}}\right)\right]-i \int_{t_{i}}^{t_{f}} \mathrm{~d} \tau \frac{1}{r_{>}}\right\}
\end{aligned}
$$

The combination of Equations (56), (60) gives the angular decomposition of the 3D Helium atom. Now we derive the sign solved propagator of the expression (60). We set

$$
\begin{array}{r}
H=\frac{p_{1}^{2}}{2}+\frac{q_{1}\left(q_{1}+1\right)}{2 r_{1}^{2}}-\frac{Z}{r_{1}} \\
H^{\prime}=\frac{p_{2}^{2}}{2}+\frac{q_{2}\left(q_{2}+1\right)}{2 r_{2}^{2}}-\frac{Z}{r_{2}}
\end{array}
$$

and

$$
H_{I}=\frac{1}{r_{>}}
$$

according to the previous section

$$
\begin{aligned}
& K_{l q_{1} q_{2}}\left(r_{1 f}, r_{2 f}, t_{f} ; r_{1 i}, r_{2 i}, t_{i}\right) \\
& =\delta\left(r_{1 f}-r_{1 i}\right) \delta\left(r_{2 f}-r_{2 i}\right) \exp \left[-i\left\langle H+H^{\prime}\right\rangle\left(t_{f}-t_{i}\right)\right] \\
& \times J_{l}\left(\frac{t_{f}-t_{i}}{\sqrt{r_{1 f}^{2}+r_{2 f}^{2}}}, \alpha_{f}\right) \exp \left(-i \frac{1}{r_{>f}}\left(t_{f}-t_{i}\right)\right)
\end{aligned}
$$

The phase in Equation (64) is calculated with respect to an appropriate sampling function. Then due to the Coulomb degeneracy of the energy of hydrogen-like atoms, we conclude that the phase in (64) is independent of the quantum numbers $q_{1}$ and $q_{2}$ and so the expectation value is constant. So within a ray theory, we can ignore it. Therefore we obtain the following expression for the SSP of the Helium atom

$$
\begin{aligned}
& K_{1}\left(\vec{r}_{1 f}, \vec{r}_{2 f}, t_{f} ; \vec{r}_{1 i}, \vec{r}_{2 i}, t_{i}\right) \\
& =\frac{4 \pi}{r_{1 f} r_{1 i} r_{2 f} r_{2 i}} \delta\left(r_{1 f}-r_{1 i}\right) \delta\left(r_{2 f}-r_{2 i}\right) \exp \left(-i \frac{1}{r_{>f}}\left(t_{f}-t_{i}\right)\right) \\
& \times h\left[r_{1 f} ; r_{2 f}\right] \sum_{l=0}^{\infty} \sum_{p=-l}^{l} \sum_{q_{1}=0}^{\infty} \sum_{m_{1}=-q_{1}}^{q_{1}} \sum_{q_{2}=0}^{\infty} \sum_{m_{2}=-q_{2}}^{q_{2}} J_{l}\left(\frac{t_{f}-t_{i}}{\sqrt{r_{1 f}^{2}+r_{2 f}^{2}}}, \alpha_{f}\right) i^{l} Y_{q_{1} m_{1}}\left(\vartheta_{1 f}, \varphi_{1 f}\right) \\
& \times Y_{l p}\left(\vartheta_{1 f}, \varphi_{1 f}\right) Y_{q_{2} m_{2}}\left(\vartheta_{2 f}, \varphi_{2 f}\right) Y_{l p}^{*}\left(\vartheta_{2 f}, \varphi_{2 f}\right) Y_{q_{1} m_{1}}^{*}\left(\vartheta_{1 i}, \varphi_{1 i}\right) Y_{q_{2} m_{2}}^{*}\left(\vartheta_{2 i}, \varphi_{2 i}\right)
\end{aligned}
$$

In the above equation, we have inserted the influence functional $h\left[r(\tau) ; r^{\prime}(\tau)\right]$ appearing in Section 2 as well. See Equation (4) as well as Equation (43). It appears in a diagonal form. For instance, in the case of a quantum dot it may correspond to the influence function of a background field. Further, the above propagator can be used in problems concerning the interaction of the atomic Helium with photonic fields. 


\section{The 2D Helium Path Integral}

Now we consider the 2D Helium [13] [14]. The Hamiltonian has the form

$$
H_{H e}^{2 D}=\frac{\vec{p}_{1}^{2}}{2}+\frac{\vec{p}_{2}^{2}}{2}-\frac{Z}{\left|\vec{\rho}_{1}\right|}-\frac{Z}{\left|\vec{\rho}_{2}\right|}+\frac{1}{\left|\vec{\rho}_{1}-\vec{\rho}_{2}\right|}
$$

where $Z$ is the atomic number. $\vec{\rho}_{1}$ and $\vec{\rho}_{2}$ are the positions of the first and the second electron respectively, with respect to the nucleus placed at the origin. Moreover $\vec{p}_{1}$ and $\vec{p}_{2}$ are their corresponding momentums.

The corresponding path integral in its discrete form is

$$
\begin{aligned}
& \tilde{K}\left(\vec{\rho}_{1 f}, \vec{\rho}_{2 f}, t_{f} ; \vec{\rho}_{1 i}, \vec{\rho}_{2 i}, t_{i}\right) \\
& =\lim _{N \rightarrow \infty} \prod_{n=1}^{N} \int_{-\infty}^{\infty} \mathrm{d} \vec{\rho}_{1 n} \prod_{n=1}^{N+1}\left[\int_{-\infty}^{\infty} \frac{\mathrm{d} \vec{p}_{1 n}}{(2 \pi)^{2}}\right] \prod_{n=1}^{N} \int_{-\infty}^{\infty} \mathrm{d} \vec{\rho}_{2 n} \prod_{n=1}^{N+1}\left[\int_{-\infty}^{\infty} \frac{\mathrm{d} \vec{p}_{2 n}}{(2 \pi)^{2}}\right] \\
& \quad \times \exp \left\{i \sum _ { n = 1 } ^ { N + 1 } \left[\vec{p}_{1 n} \cdot\left(\vec{\rho}_{1 n}-\vec{\rho}_{1 n-1}\right)+\vec{p}_{2 n} \cdot\left(\vec{\rho}_{2 n}-\vec{\rho}_{2 n-1}\right)\right.\right. \\
& \left.\left.-\varepsilon\left(\frac{\vec{p}_{1 n}^{2}}{2}+\frac{\vec{p}_{2 n}^{2}}{2}-\frac{Z}{\left|\vec{\rho}_{1 n}\right|}-\frac{Z}{\left|\vec{\rho}_{2 n}\right|}+\frac{1}{\left|\vec{\rho}_{1 n}-\vec{\rho}_{2 n}\right|}\right)\right]\right\}
\end{aligned}
$$

where $\varepsilon=\frac{t_{f}-t_{i}}{N+1}$. We have also set $\vec{\rho}_{10}=\vec{\rho}_{1 i}, \quad \vec{\rho}_{20}=\vec{\rho}_{2 i}, \quad \vec{\rho}_{1 N+1}=\vec{\rho}_{1 f}$ and $\vec{\rho}_{2 N+1}=\vec{\rho}_{2 f}$. Now we observe that the two electrons' interaction term can be written as

$$
\begin{aligned}
& \frac{1}{\left|\vec{\rho}_{1 n}-\vec{\rho}_{2 n}\right|}=\frac{1}{\sqrt{\rho_{1 n}^{2}+\rho_{2 n}^{2}-2 \vec{\rho}_{1 n} \cdot \vec{\rho}_{2 n}}}=\frac{1}{\sqrt{\rho_{1 n}^{2}+\rho_{2 n}^{2}-2 \rho_{1 n} \rho_{2 n} \cos \varphi_{12 n}}} \\
& =\frac{1}{\sqrt{\rho_{1 n}^{2}+\rho_{2 n}^{2}}} \frac{1}{\sqrt{1-\frac{2 \rho_{1 n} \rho_{2 n}}{\rho_{1 n}^{2}+\rho_{2 n}^{2}} \cos \varphi_{12 n}}} \\
& =\frac{1}{\sqrt{\rho_{1 n}^{2}+\rho_{2 n}^{2}}} \frac{1}{\sqrt{1-\frac{2}{\frac{\rho_{1 n}}{\rho_{2 n}}+\frac{\rho_{2 n}}{\rho_{1 n}}} \cos \varphi_{12 n}}} \\
& =\frac{1}{\sqrt{\rho_{1 n}^{2}+\rho_{2 n}^{2}}} \frac{1}{\sqrt{1-\alpha\left(\rho_{1 n}, \rho_{2 n}\right) \cos \varphi_{12 n}}}
\end{aligned}
$$

In the last equality, we have set

$$
\alpha\left(\rho_{1 n}, \rho_{2 n}\right)=\frac{2}{\frac{\rho_{1 n}}{\rho_{2 n}}+\frac{\rho_{2 n}}{\rho_{1 n}}} \leq 1
$$

Moreover $\rho_{1 n}=\left|\vec{\rho}_{1 n}\right|, \quad \rho_{2 n}=\left|\vec{\rho}_{2 n}\right|$. Then the path integral (67) takes the form

$$
\begin{aligned}
& \tilde{K}\left(\vec{\rho}_{1 f}, \vec{\rho}_{2 f}, t_{f} ; \vec{\rho}_{1 i}, \vec{\rho}_{2 i}, t_{i}\right) \\
& =\lim _{N \rightarrow \infty} \prod_{n=1}^{N} \int_{-\infty}^{\infty} \mathrm{d} \vec{\rho}_{1 n} \prod_{n=1}^{N+1}\left[\int_{-\infty}^{\infty} \frac{\mathrm{d} \vec{p}_{1 n}}{(2 \pi)^{2}}\right] \prod_{n=1}^{N} \int_{-\infty}^{\infty} \mathrm{d} \vec{\rho}_{2 n} \prod_{n=1}^{N+1}\left[\int_{-\infty}^{\infty} \frac{\mathrm{d} \vec{p}_{2 n}}{(2 \pi)^{2}}\right]
\end{aligned}
$$




$$
\begin{aligned}
& \times \exp \left\{i \sum _ { n = 1 } ^ { N + 1 } \left[\vec{p}_{1 n} \cdot\left(\vec{\rho}_{1 n}-\vec{\rho}_{1 n-1}\right)+\vec{p}_{2 n} \cdot\left(\vec{\rho}_{2 n}-\vec{\rho}_{2 n-1}\right)\right.\right. \\
& \left.\left.-\varepsilon\left(\frac{\vec{p}_{1 n}^{2}}{2}+\frac{\vec{p}_{2 n}^{2}}{2}-\frac{Z}{\rho_{1 n}}-\frac{Z}{\rho_{2 n}}+\frac{1}{\sqrt{\rho_{1 n}^{2}+\rho_{2 n}^{2}}} \frac{1}{\sqrt{1-\alpha\left(\rho_{1 n}, \rho_{2 n}\right) \cos \varphi_{12 n}}}\right)\right]\right\}
\end{aligned}
$$

To proceed we insert in Equation (70) a delta function and get

$$
\begin{aligned}
& \tilde{K}\left(\vec{\rho}_{1 f}, \vec{\rho}_{2 f}, t_{f} ; \vec{\rho}_{1 i}, \vec{\rho}_{2 i}, t_{i}\right) \\
& =\lim _{N \rightarrow \infty} \prod_{n=1}^{N} \int_{-\infty}^{\infty} \mathrm{d} \vec{\rho}_{1 n} \prod_{n=1}^{N+1}\left[\int_{-\infty}^{\infty} \frac{\mathrm{d} \vec{p}_{1 n}}{(2 \pi)^{2}}\right] \prod_{n=1}^{N} \int_{-\infty}^{\infty} \mathrm{d} \vec{\rho}_{2 n} \prod_{n=1}^{N+1}\left[\int_{-\infty}^{\infty} \frac{\mathrm{d} \vec{p}_{2 n}}{(2 \pi)^{2}}\right] \\
& \times \prod_{n=1}^{N+1}\left[\int_{-\infty}^{\infty} \mathrm{d} w_{n}\right] \prod_{n=1}^{N+1}\left[\delta\left(w_{n}-\alpha\left(\rho_{1 n}, \rho_{2 n}\right) \cos \varphi_{12 n}\right)\right] \\
& \times \exp \left\{i \sum _ { n = 1 } ^ { N + 1 } \left[\vec{p}_{1 n} \cdot\left(\vec{\rho}_{1 n}-\vec{\rho}_{1 n-1}\right)+\vec{p}_{2 n} \cdot\left(\vec{\rho}_{2 n}-\vec{\rho}_{2 n-1}\right)\right.\right. \\
& \left.\left.-\varepsilon\left(\frac{\vec{p}_{1 n}^{2}}{2}+\frac{\vec{p}_{2 n}^{2}}{2}-\frac{Z}{\rho_{1 n}}-\frac{Z}{\rho_{2 n}}+\frac{1}{\sqrt{\rho_{1 n}^{2}+\rho_{2 n}^{2}}} \frac{1}{\sqrt{1-w_{n}}}\right)\right]\right\}
\end{aligned}
$$

Now the delta functions in Equation (71) have the representation

$$
\begin{aligned}
& \delta\left(w_{n}-\alpha_{n} \cos \varphi_{12 n}\right)=\frac{1}{2 \pi} \int_{-\infty}^{\infty} \mathrm{d} \lambda_{n} \mathrm{e}^{-i \lambda_{n} w_{n}} \mathrm{e}^{i \lambda \lambda_{n} \alpha_{n} \cos \varphi_{12 n}} \\
& =\frac{1}{2 \pi} \int_{-\infty}^{\infty} \mathrm{d} \lambda_{n} \mathrm{e}^{-i \lambda_{n} w_{n}} J_{0}\left(\lambda_{n} \alpha_{n}\right)+\frac{1}{\pi} \sum_{k_{n}=1}^{\infty} i^{k_{n}} \cos \left(k_{n} \varphi_{12 n}\right) \int_{-\infty}^{\infty} \mathrm{d} \lambda_{n} \mathrm{e}^{-i \lambda_{n} w_{n}} J_{k_{n}}\left(\lambda_{n} \alpha_{n}\right) \\
& =\frac{1}{\pi} \int_{0}^{\infty} \mathrm{d} \lambda_{n} \cos \left(\lambda_{n} w_{n}\right) J_{0}\left(\lambda_{n} \alpha_{n}\right) \\
& +\frac{1}{\pi} \sum_{k_{n}=1}^{\infty} i^{k_{n}} \cos \left(k_{n} \varphi_{12 n}\right) \int_{0}^{\infty} \mathrm{d} \lambda_{n}\left[\mathrm{e}^{-i \lambda_{n} w_{n}}+(-1)^{k_{n}} \mathrm{e}^{i \lambda_{n} w_{n}}\right] J_{k_{n}}\left(\lambda_{n} \alpha_{n}\right)
\end{aligned}
$$

where $\alpha_{n}=\alpha\left(\rho_{1 n}, \rho_{2 n}\right)$. Moreover, the integrals appearing in Equation (72) are given by the expressions

$$
\begin{gathered}
f_{2 k}^{c}(w, \alpha)=\int_{0}^{\infty} \mathrm{d} \lambda \cos (\lambda w) J_{2 k}(\lambda \alpha)= \begin{cases}\frac{\cos \left[2 k \arcsin \left(\frac{w}{\alpha}\right)\right]}{\sqrt{\alpha^{2}-w^{2}}} & |w|<\alpha \quad(73) \\
0 & |w|>\alpha\end{cases} \\
f_{2 k+1}^{s}(w, \alpha)=\int_{0}^{\infty} \mathrm{d} \lambda \sin (\lambda w) J_{2 k+1}(\lambda \alpha)= \begin{cases}\frac{\sin \left[(2 k+1) \arcsin \left(\frac{w}{\alpha}\right)\right]}{\sqrt{\alpha^{2}-w^{2}}} & |w|<\alpha(74) \\
0 & |w|>\alpha\end{cases}
\end{gathered}
$$

If $k=0$ Equation (73) becomes

$$
f_{0}^{c}(w, \alpha)=\int_{0}^{\infty} \mathrm{d} \lambda \cos (\lambda w) J_{0}(\lambda \alpha)= \begin{cases}\frac{1}{\sqrt{\alpha^{2}-w^{2}}} & |w|<\alpha \\ 0 & |w|>\alpha\end{cases}
$$


Therefore we can perform the integrations in Equation (72) according to the expressions (73)-(75) and place the results in Equation (71) after keeping leading terms with respect the $k_{n}$ 's as after the angular decomposition the angular parts of higher-order terms in the expansion (72) contribute infinities as $N \rightarrow \infty$. So Equation (71) becomes

$$
\begin{aligned}
& \tilde{K}\left(\vec{\rho}_{1 f}, \vec{\rho}_{2 f}, t_{f} ; \vec{\rho}_{1 i}, \vec{\rho}_{2 i}, t_{i}\right) \\
& =\lim _{N \rightarrow \infty} \prod_{n=1}^{N} \int_{-\infty}^{\infty} \mathrm{d} \vec{\rho}_{1 n} \prod_{n=1}^{N+1}\left[\int_{-\infty}^{\infty} \frac{\mathrm{d} \vec{p}_{1 n}}{(2 \pi)^{2}}\right] \prod_{n=1}^{N} \int_{-\infty}^{\infty} \mathrm{d} \vec{\rho}_{2 n} \prod_{n=1}^{N+1}\left[\int_{-\infty}^{\infty} \frac{\mathrm{d} \vec{p}_{2 n}}{(2 \pi)^{2}}\right] \prod_{n=1}^{N+1}\left[\int_{-\alpha_{n}}^{\alpha_{n}} \frac{\mathrm{d} w_{n}}{\pi}\right] \prod_{n=1}^{N}\left[f_{0}^{c}\left(w_{n}, \alpha_{n}\right)\right] \\
& \times\left(f_{0}^{c}\left(w_{N+1}, \alpha_{N+1}\right)+2 \sum_{k_{N+1}=1}^{\infty}(-1)^{k_{N+1}} \cos \left[2 k_{N+1} \varphi_{12 N+1}\right] f_{2 k_{N+1}}^{c}\left(w_{N+1}, \alpha_{N+1}\right)\right. \\
& \left.+2 \sum_{k_{N+1}=0}^{\infty}(-1)^{k_{N+1}} \cos \left[\left(2 k_{N+1}+1\right) \varphi_{12 N+1}\right] f_{2 k_{N+1}+1}^{s}\left(w_{N+1}, \alpha_{N+1}\right)\right) \\
& \times \exp \left\{i \sum _ { n = 1 } ^ { N + 1 } \left[\vec{p}_{1 n} \cdot\left(\vec{\rho}_{1 n}-\vec{\rho}_{1 n-1}\right)+\vec{p}_{2 n} \cdot\left(\vec{\rho}_{2 n}-\vec{\rho}_{2 n-1}\right)\right.\right. \\
& \left.\left.-\varepsilon\left(\frac{\vec{p}_{1 n}^{2}}{2}+\frac{\vec{p}_{2 n}^{2}}{2}-\frac{Z}{\rho_{1 n}}-\frac{Z}{\rho_{2 n}}+\frac{1}{\sqrt{\rho_{1 n}^{2}+\rho_{2 n}^{2}}} \frac{1}{\sqrt{1-w_{n}}}\right)\right]\right\}
\end{aligned}
$$

We have set the range of $w_{n}$ in the interval from $-\alpha_{n}$ to $\alpha_{n}$ as otherwise, the functions $f_{2 k}^{c}$ and $f_{2 k+1}^{s}$ are zero (see Equations (73)-(75)). We notice that in the case of the $N+1$ factor appearing in Equation (75) we have kept the full series (72) as it involves the final coordinates.

Now in the two-dimensional path integral (76) we perform standard manipulations including an angular decomposition to get

$$
\begin{aligned}
& \tilde{K}\left(\vec{\rho}_{1 f}, \vec{\rho}_{2 f}, t_{f} ; \vec{\rho}_{1 i}, \vec{\rho}_{2 i}, t_{i}\right) \\
& =\frac{1}{\sqrt{\rho_{1 f} \rho_{1 i} \rho_{2 f} \rho_{2 i}}} \sum_{q_{1}=-\infty}^{\infty} \sum_{q_{2}=-\infty}^{\infty} \frac{1}{2 \pi} \mathrm{e}^{i q_{1}\left(\varphi_{1 f}-\varphi_{1 i}\right)} \frac{1}{2 \pi} \mathrm{e}^{i q_{2}\left(\varphi_{2 f}-\varphi_{2 i}\right)}\left[\tilde{K}_{0 q_{1} q_{2}}^{c}\left(\rho_{1 f}, \rho_{2 f}, t_{f} ; \rho_{1 i}, \rho_{2 i}, t_{i}\right)\right. \\
& +\sum_{k=1}^{\infty}(-1)^{k}\left(\mathrm{e}^{2 i k\left(\varphi_{1 f}-\varphi_{2 f}\right)}+\mathrm{e}^{-2 i k\left(\varphi_{1 f}-\varphi_{2 f}\right)}\right) \tilde{K}_{2 k q_{1} q_{2}}^{c}\left(\rho_{1 f}, \rho_{2 f}, t_{f} ; \rho_{1 i}, \rho_{2 i}, t_{i}\right) \\
& \left.+\sum_{k=0}^{\infty}(-1)^{k}\left(\mathrm{e}^{i(2 k+1)\left(\varphi_{1 f}-\varphi_{2 f}\right)}+\mathrm{e}^{-i(2 k+1)\left(\varphi_{1 f}-\varphi_{2 f}\right)}\right) \tilde{K}_{2 k+1 q_{1} q_{2}}^{s}\left(\rho_{1 f}, \rho_{2 f}, t_{f} ; \rho_{1 i}, \rho_{2 i}, t_{i}\right)\right]
\end{aligned}
$$

$q_{1}$ and $q_{2}$ have appeared after the angular decomposition and are the azimuthal quantum numbers corresponding to electrons 1 and 2 respectively. The $\tilde{K}_{\left\{\begin{array}{l}\{c \\ s\} \\ 2 k+1\end{array}\right\}}^{\substack{c k \\ 2 k \\ q_{1} q_{2}}}\left(\rho_{1 f}, \rho_{2 f}, t_{f} ; \rho_{1 i}, \rho_{2 i}, t_{i}\right)$ terms above correspond to the path integral

$$
\begin{aligned}
& \tilde{K}_{\{2 k}^{\left\{\begin{array}{c}
c \\
s\} \\
2 k+1\}
\end{array}\right\} q_{1} q_{2}}\left(\rho_{1 f}, \rho_{2 f}, t_{f} ; \rho_{1 i}, \rho_{2 i}, t_{i}\right) \\
& =\lim _{N \rightarrow \infty} \prod_{n=1}^{N} \int_{0}^{\infty} \mathrm{d} \rho_{1 n} \prod_{n=1}^{N+1}\left[\int_{-\infty}^{\infty} \frac{\mathrm{d} p_{1 n}}{2 \pi}\right] \prod_{n=1}^{N} \int_{0}^{\infty} \mathrm{d} \rho_{2 n} \prod_{n=1}^{N+1}\left[\int_{-\infty}^{\infty} \frac{\mathrm{d} p_{2 n}}{2 \pi}\right] \\
& \times \exp \left\{i \sum _ { n = 1 } ^ { N + 1 } \left[p_{1 n}\left(\rho_{1 n}-\rho_{1 n-1}\right)+p_{2 n}\left(\rho_{2 n}-\rho_{2 n-1}\right)\right.\right.
\end{aligned}
$$




$$
\begin{aligned}
& \left.\left.-\varepsilon\left(\frac{p_{1 n}^{2}}{2}+\frac{q_{1}^{2}-\frac{1}{4}}{2 \rho_{1 n}^{2}}-\frac{Z}{\rho_{1 n}}+\frac{p_{2 n}^{2}}{2}+\frac{q_{2}^{2}-\frac{1}{4}}{2 \rho_{2 n}^{2}}-\frac{Z}{\rho_{2 n}}\right)\right]\right\}
\end{aligned}
$$

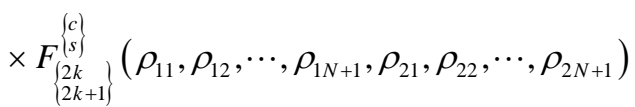

We have set $\rho_{10}=\rho_{1 i}, \quad \rho_{20}=\rho_{2 i}, \quad \rho_{1 N+1}=\rho_{1 f}$ and $\rho_{2 N+1}=\rho_{2 f}$ while the fac-

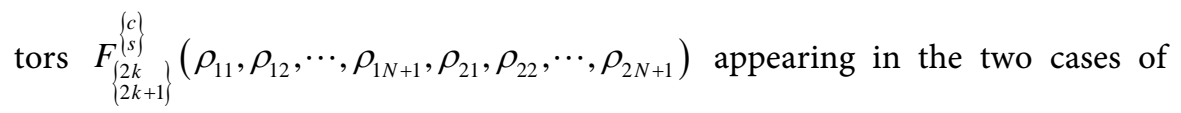
Equation (78) above has the form

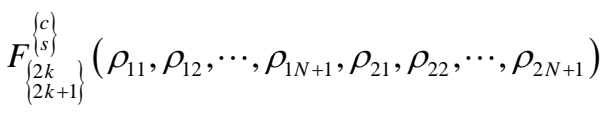

$$
\begin{aligned}
& =\prod_{n=1}^{N+1}\left[\int_{-\alpha_{n}}^{\alpha_{n}} \frac{\mathrm{d} w_{n}}{\pi}\right] \prod_{n=1}^{N}\left[f_{0}^{c}\left(w_{n}, \alpha_{n}\right)\right] f_{\left\{\begin{array}{c}
(s) \\
(s) \\
2 k+1\}
\end{array}\right\}}^{\substack{s \\
k}}\left(w_{N+1}, \alpha_{N+1}\right) \\
& \times \exp \left\{-i \varepsilon \sum_{n=1}^{N+1}\left[\frac{1}{\sqrt{\rho_{1 n}^{2}+\rho_{2 n}^{2}}} \frac{1}{\sqrt{1-w_{n}}}\right]\right\}
\end{aligned}
$$

We observe that the above multiple integral can be decomposed in $N+1$ one-dimensional integrals.

Now we solve the sign problem. The following inequality is valid

$$
\left.\mid \begin{array}{c}
\left\{\begin{array}{c}
c \\
c \\
F_{2 k}^{(s)}\{ \\
2 k+1
\end{array}\right\} \\
2 k
\end{array}\right\}\left(\rho_{11}, \rho_{12}, \cdots, \rho_{1 N+1}, \rho_{21}, \rho_{22}, \cdots, \rho_{2 N+1}\right) \mid \leq b
$$

where $b$ is a positive constant. Therefore the sign solved propagator theorem is applicable and the corresponding sign solved propagators are

$$
\begin{aligned}
& \tilde{K}_{\{2 k}^{\substack{c c \\
\{k+1\} \\
2 k\}}} q_{1 q_{2} q_{2}}\left(\rho_{1 f}, \rho_{2 f}, t_{f} ; \rho_{1 i}, \rho_{2 i}, t_{i}\right) \\
& =\delta\left(\rho_{1 f}-\rho_{1 i}\right) \delta\left(\rho_{2 f}-\rho_{2 i}\right) \exp \left\{-i\left\langle H_{0}^{q_{1} q_{2}}\right\rangle\left(t_{f}-t_{i}\right)\right\}
\end{aligned}
$$

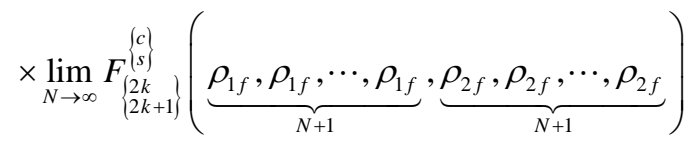

In Equation (81) we have set

$$
H_{0}^{q_{1} q_{2}}=\frac{p_{1}^{2}}{2}+\frac{q_{1}^{2}-\frac{1}{4}}{2 \rho_{1}^{2}}-\frac{Z}{\rho_{1}}+\frac{p_{2}^{2}}{2}+\frac{q_{2}^{2}-\frac{1}{4}}{2 \rho_{2}^{2}}-\frac{Z}{\rho_{2}}
$$

An appropriate sampling function is used in the evaluation of the expectation value of $H_{0}^{q_{1} q_{2}}$ on the phase in Equation (81). However, in most of the final results of interest, that phase does not appear. Eventually

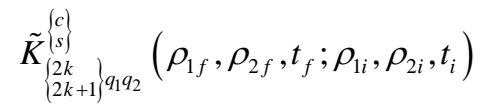

$$
\begin{aligned}
& =\delta\left(\rho_{1 f}-\rho_{1 i}\right) \delta\left(\rho_{2 f}-\rho_{2 i}\right) h\left[\rho_{1 f} ; \rho_{2 f}\right] \exp \left(-i\left\langle H_{0}^{q_{1} q_{2}}\right\rangle\left(t_{f}-t_{i}\right)\right)
\end{aligned}
$$




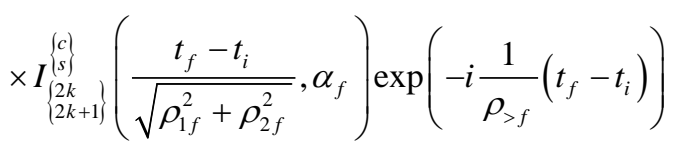

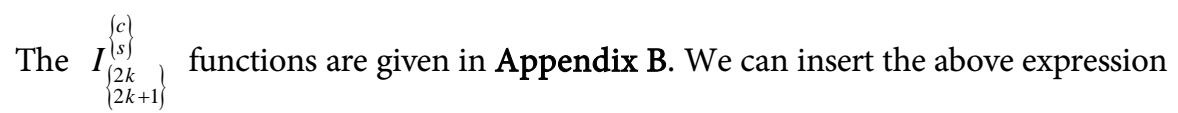
in Equation (77). As in Equation (65) a possible influence functional $h\left[\rho_{1 f} ; \rho_{2 f}\right]$ has been inserted and appears in a diagonal form. It may correspond to the effect of a background field in a $2 \mathrm{D}$ quantum dot.

Another application is the use of the present SSP in the evaluation of the geometric phase of the 2D Helium [14].

\section{Conclusions}

In the present paper, we use the central limit theorem to the solution of the sign problem and the extraction of the SSP in the case of a double phase space path integral corresponding to two interacting particles in a potential.

Further, in order to apply that theory, we consider the $3 \mathrm{D}$ and $2 \mathrm{D}$ Helium atoms. We perform the angular decomposition of their path integrals and via the present theory, we extract their SSP expressions.

Concluding the present paper's methods based on the use of the central limit theorem are tractable and can be used in atomic physics problems or in the study of quantum dots.

\section{Conflicts of Interest}

The author declares no conflicts of interest regarding the publication of this paper.

\section{References}

[1] Meerschaert, M.M. and Scheffler, H.-P. (2001) Limit Distributions for Sums of Independent Random Vectors. John Wiley \& Sons, Inc., New York.

[2] Port, S.C. (1994) Theoretical Probability for Applications. John Wiley \& Sons, Inc., New York.

[3] Gnedenko, B.V. and Kolmogorov, A.N. (1968) Limit Distributions for Sums of Independent Random Variables, Addison-Wesley Series in Statistics, Addison-Wesley, Boston.

[4] Thrapsaniotis, E.G. (2003) Monte Carlo Methods via the Use of the Central-Limit Theorem. Europhysics Letters, 63, 479. https://doi.org/10.1209/epl/i2003-00491-5

[5] Thrapsaniotis, E.G. (2008) Path Integral Methods via the Use of the Central Limit Theorem and Application. Journal of Physics A: Mathematical and Theoretical, 41, Article No. 205202. https://doi.org/10.1088/1751-8113/41/20/205202

[6] Thrapsaniotis, E.G. (2007) Coherent State Path Integral Methods and Central Limit Theorem. Physics Letters A, 365, 191-199. https://doi.org/10.1016/j.physleta.2007.01.011

[7] Makri, Nancy (1995) Numerical Path Integral Techniques for Long Time Dynamics of Quantum Dissipative Systems. Journal of Mathematical Physics, 36, 2430. https://doi.org/10.1063/1.531046 
[8] Kleinert, H. (2009) Path Integrals in Quantum Mechanics, Statistics, Polymer Physics, and Financial Markets. World Scientific, Singapore. https://doi.org/10.1142/7305

[9] Allmendinger, T. (2018) The Atom Model of Helium and of Neon Based on the Theorem of Niels Bohr. Journal of Applied Mathematics and Physics, 6, 1290-1300. https://doi.org/10.4236/jamp.2018.66108

[10] Friedrich, H. (2006) Theoretical Atomic Physics. Springer, Berlin.

[11] Bethe, H.A. and Salpeter, E.E. (2008) Quantum Mechanics of One- and Two-Electron Atoms. Dover, New York.

[12] Eyges, L. (1959) Quantum-Mechanical Three-Body Problem. Physical Review, 115, Article No. 1643. https://doi.org/10.1103/PhysRev.115.1643

[13] Hilico, L., Gremaud, B., Jonckheere, T., Billy, N. and Delande, D. (2002) Quantum Three-Body Coulomb Problem in Two Dimensions. Physical Review A, 66, Article No. 022101. https://doi.org/10.1103/PhysRevA.66.022101

[14] Thrapsaniotis, E.G. (2010) Sign Solved Propagator of a Two Electrons 2D Atom and Application. Far East Journal of Dynamical Systems, 14, 171-182. 


\section{Appendix A: Integrals}

In the equations of Section 3 there appear integrals of the following form (see Equations (51 to 54) for a definition of the $g_{l}(u)$ )

$$
J_{l}^{0}(b, u)=\int \mathrm{d} u g_{l}(u) \exp \left[-i \frac{b}{\sqrt{1-u}}\right]
$$

After standard calculations we obtain

$$
\begin{aligned}
J_{0}^{0}(b, u)= & \frac{1}{2 \alpha}(u-1+i b \sqrt{1-u}) \exp \left[-i \frac{b}{\sqrt{1-u}}\right]-\frac{b^{2}}{2 \alpha} E i\left[-i \frac{b}{\sqrt{1-u}}\right] \\
J_{1}^{0}(b, u)= & -\frac{1}{24 \alpha^{2}} i\left(i b^{3} \sqrt{1-u}+b^{2}(u-1)+2 i b \sqrt{1-u}(u+5)+6\left(u^{2}-1\right)\right) \\
& \times \exp \left[-i \frac{b}{\sqrt{1-u}}\right]+\frac{1}{24 \alpha^{2}} i b^{2}\left(12+b^{2}\right) E i\left[-i \frac{b}{\sqrt{1-u}}\right] \\
J_{2}^{0}(b, u)= & \frac{1}{480 \alpha^{3}}\left(-i b^{5} \sqrt{1-u}-b^{4}(u-1)-6 b^{2}(u-1)(u+9)\right. \\
& -2 i b^{3} \sqrt{1-u}(u+29)+120 \alpha^{2}(u-1+i b \sqrt{1-u})-120\left(u^{3}-1\right) \\
& \left.-24 i b \sqrt{1-u}\left(u^{2}+3 u+11\right)\right) \exp \left[-i \frac{b}{\sqrt{1-u}}\right] \\
& +\frac{1}{480 \alpha^{3}} b^{2}\left(360-120 \alpha^{2}+60 b^{2}+b^{4}\right) E i\left[-i \frac{b}{\sqrt{1-u}}\right]
\end{aligned}
$$

The functions $J_{l}$ in Equations (60), (64), (65) have the form

$$
J_{l}(b, \alpha)=J_{l}^{0}(b, \alpha)-J_{l}^{0}(b,-\alpha)
$$

where $b=\frac{t_{f}-t_{i}}{\sqrt{r_{1 f}^{2}+r_{2 f}^{2}}}$. Further $\sqrt{1-\alpha}=\frac{\left|r_{1}-r_{2}\right|}{\sqrt{r_{1}^{2}+r_{2}^{2}}}$ and $\sqrt{1+\alpha}=\frac{r_{1}+r_{2}}{\sqrt{r_{1}^{2}+r_{2}^{2}}}$.

\section{Appendix B: Integrals}

In Section 4 there appear integrals of the forms

$$
\begin{aligned}
& I_{2 k}^{c}(b, \alpha)=\sum_{n=0}^{\infty} \frac{(-i b)^{n}}{n !} \int_{-\pi / 2}^{\pi / 2} \frac{\mathrm{d} \theta}{\pi} \frac{\cos [2 k \theta]}{(1-\alpha \sin (\theta))^{n / 2}} \\
& I_{2 k+1}^{s}(b, \alpha)=\sum_{n=0}^{\infty} \frac{(-i b)^{n}}{n !} \int_{-\pi / 2}^{\pi / 2} \frac{\mathrm{d} \theta}{\pi} \frac{\sin [(2 k+1) \theta]}{(1-\alpha \sin (\theta))^{n / 2}}
\end{aligned}
$$

where $b=\frac{t_{f}-t_{i}}{\sqrt{\rho_{1 f}^{2}+\rho_{2 f}^{2}}}$. Therefore

$$
\begin{gathered}
I_{0}^{c}=\sum_{n=0}^{\infty} \frac{(-i b)^{n}}{n !}{ }_{2} F_{1}\left(\frac{n}{4}+\frac{1}{2}, \frac{n}{4} ; 1 ; \alpha^{2}\right) \\
I_{1}^{s}=\frac{\alpha}{4} \sum_{n=1}^{\infty} \frac{(-i b)^{n}}{(n-1) !}{ }_{2} F_{1}\left(\frac{n}{4}+\frac{1}{2}, \frac{n}{4}+1 ; 2 ; \alpha^{2}\right)
\end{gathered}
$$




$$
\begin{gathered}
I_{2}^{c}=\sum_{n=0}^{\infty} \frac{(-i b)^{n}}{n !}\left[{ }_{2} F_{1}\left(\frac{n}{4}, \frac{n}{4}+\frac{1}{2} ; 1 ; \alpha^{2}\right)-{ }_{3} F_{2}\left(\frac{3}{2}, \frac{n}{4}+\frac{1}{2}, \frac{n}{4} ; \frac{1}{2}, 2 ; \alpha^{2}\right)\right] \\
I_{3}^{s}=\frac{3}{4} \alpha \sum_{n=1}^{\infty} \frac{(-i b)^{n}}{(n-1) !}\left[{ }_{2} F_{1}\left(\frac{n}{4}+\frac{1}{2}, \frac{n}{4}+1 ; 2 ; \alpha^{2}\right)-{ }_{3} F_{2}\left(\frac{5}{2}, \frac{n}{4}+\frac{1}{2}, \frac{n}{4}+1 ; \frac{3}{2}, 3 ; \alpha^{2}\right)\right]
\end{gathered}
$$

